\title{
Profile of Injuries Among Sitting Volleyball Players with Disabilities in Rwanda
}

\author{
Assuman Nuhu ${ }^{1 *}$, Ben Honoré Nishimwe ${ }^{1}$, Jean Bosco Nteziryayo ${ }^{1}$, Aimée Flora Nyirahabimana ${ }^{1}$, Jackline \\ Starehe ${ }^{1}$, Anne Kumurenzi ${ }^{1}$, Jean Baptiste Sagahutu ${ }^{1}$ \\ ${ }^{1}$ Department of Physiotherapy, College of Medicine and Health Sciences,University of Rwanda
}

*Corresponding author: Assuman Nuhu. Department of Physiotherapy, College of Medicine and Health Sciences, University of Rwanda, Remera Campus, KG 11 Ave, 47, Kigali, Rwanda.Email:nuhu.assuman@gmail.com

\begin{abstract}
Background

Sports injuries are some of the risk of paralympics sport. Injuries are somewhat high compared with injury rates in able-bodied athletes. Injuries might have an impact on the participation and future health. It is paramount to investigate sports specific injuries to eliminate associated risks. Aim

To identify patterns of injuries among sitting volleyball players in Rwanda.

Methods

A prospective quantitative study was conducted among 158 sitting volleyball players competing in the 2013-2014 season. The research team composed by physiotherapists used the data collection process following the consensus on injury definitions and data collection procedures. The ethical clearance and permission were obtained from relevant bodies. ANOVA or the Mann-Whitney U-test were used for continuous variables while Chi-square or Fisher's exact tests were used for categorical variables.

\section{Results}

All the 158 players aged $25 \pm 6$ years participated in the study; with male predominance $(66.5 \%)$. Fifty-eight players $(37 \%)$ sustained at least one injury. Nearly half of injuries $(n=43)$ were mild, consistent for both male and female. There was no statistical difference in the patterns of injuries comparing male and female participants.

\section{Conclusion}

Sitting volleyball players with disability in Rwanda sustained a high prevalence of injuries. The fingers, hands and wrists were the most injured body parts. However, the health and integrative advantages should outweigh the risk of adverse effects associated with participation in sports such as injuries.

Rwanda J Med Health Sci 2019;2(3):258-264.
\end{abstract}

Keywords: Sitting volleyball, People with disabilities (PWDs), Injury

\section{Introduction}

People with disabilities (PWDs) should receive the same physical, mental, and social benefits from participating in sport and physical activity as those not having a disability.[1] The number of athletes with disabilities participating in organized sports and the popularity of the games for PWDs is steadily increasing around the world. Sitting volleyball is a sport developed for athletes with lower extremity impairment for example amputations; disabling joint injuries or conditions and limb weakness.[2] To raise awareness of the rights of PWDs, Rwanda has given the opportunity to around 300 persons and about 100 youth with disabilities to access sport and recreational activities (NPC).[3] The results of the study conducted by Barayagwiza indicated that the top five sports disciplines accessed by youth with physical disabilities in Rwanda were sit ball, sitting volleyball, volleyball, table tennis and wheelchair basketball[4].
The growing interest and participation in sports increase the risk for injury among athletes with disabilities. However, there are few studies concerning injury patterns, risk factors, and prevention strategies of injuries in paralympic sports. The study conducted by Fagher and Lexell[5] showed that the overall rates of injuries are considerably high and comparable with injury rates in able-bodied athletes.

Upper limb injuries are common in sitting volleyball. The report from the study conducted among athletes in paralympic games in 2012 indicated that the most commonly injured region was the shoulder $(17.7 \%)$, followed by the wrist/hand (11.4\%) and elbow (8.8\%). [6] Furthermore, the most frequent type of injuries sustained by sitting volleyball players were sprains, dislocations of joints, and bruising of various body parts. The most common mechanisms of injuries 
were spiking or blocking the ball.[7] These injuries contribute to serious joint and muscle dysfunction in addition to their disabilities and may lead to restriction in sports participation. However, there is a scarcity of information on common injuries in disability sports in general and particularly among sitting volleyball players with disabilities in Rwanda.

This study will provide baseline information about common injuries, patterns, mechanism and predisposing factors of injury among sitting volleyball players in Rwanda. This study will also raise awareness of the participants, team managers, staff and medical team about the occurrence of injuries to propose appropriate interventions.

\section{Methods}

\section{Design}

A prospective quantitative study was conducted among Rwanda male and female sitting volleyball players to record injuries throughout the season. The competitive season was from September 2013 to March 2014.

\section{Population and sample}

The study population comprised 158 Rwandan sitting volleyball players holding a valid league participation license to play in the 2013-2014 season. All the 17 teams were included in the study. Teams registered between 8 and 10 players ( 3 teams registered 8 players, 6 teams had 9 players and 8 teams had 10 players). The study included all the players participating in the league. We included all the 158 players that had been participating in team training practice sessions and had been actively taking part in league tournaments. Players presenting with pre-existing injuries were only included in the study once cleared to participate in training and matches. The sample size of 158 players was found to be enough to depict any significant difference in variables under investigation.

\section{Data Collection tools and procedures}

This study design followed the consensus on injury definitions and data collection procedures used in ablebodied studies of injuries.[7,8] A self-designed short form was used to collect demographic information of the players. The form included the age of the player, weight and height, playing position, playing experience, occupation and marital status. The information pertaining to the type of impairments was also included in the form. A standardised injury report form was used by the research team to collect data pertaining to the occurrence of injuries. The injury report form is comprehensive and injuries are recorded on the front side of the form, while all definitions related to the type, anatomical location and severity of the injury are provided on the back of the form. The operational definitions adopted in this study have been widely advocated for epidemiological studies in sports.[7,9] A recordable injury was the one that caused the player to be unavailable for full team participation in training or match play. All injuries were followed until the final day of rehabilitation corresponding to full training and match participation. The day on which an injury occurred was not counted when determining the severity of an injury. The severity of injuries was classified according to the days that players were unable to participate in full training or competition. An injury that caused a time loss of 1-3 days was classified as "minimal", while injuries that resulted in an absence from training or matches for 4-7, 8-21 and more than 21 days were classified as "mild", "moderate" and "severe" injuries respectively. A recurrent injury was defined as an injury of the same type and in the same location to the previously sustained injury within 2 months.

A familiarization session was organized for the research team to have a common understanding of the definitions used in the study, data collection tools and procedures. A formal research ethics approval from the University of Rwanda Institutional Review Board and appropriate permissions from the teams and participants' consent were sought. Before the beginning of the league, the research team collected information pertaining to the demographics of the participants as well as the impairments of the participants through a physical assessment. The assessment was done at the teams' convenient time. Data collection started on 8 September 2013 and ended on 29 March 2014.

\section{Measures of injury}

The research team visited each team on a weekly basis up on the start of the season to record any injury that happened in that particular week. This process was adopted because there were no medical personnel in the teams. Players were asked whether they had sustained any injury in the just ended week. If any, more data was recorded as to the date of injury, location, type and whether it is a recurrent injury. The date of return to play after injury was also recorded in the subsequent visit to account for the severity of injuries. The above information was recorded on the standardized injury report form.

\section{Data analysis}

The Statistical Package for the Social Sciences (SPSS version 16.0, SPSS Inc., Chicago, IL, USA) was used for statistical analysis. The numerical data were analyzed using descriptive statistics (mean and 95\% confidence intervals) to describe each variable. The categorical and nominal variables were analysed using frequencies and proportions presented in form of percentages. Normality test were conducted for continuous variables using Kolmogorov-Smirnov and Shapiro-Wilk tests for skewness and kurtosis. Additionally, the visual representation of the data was also done for the stemand-leaf, Q-Q plot and histogramme. The variables 
age, weight and height were not normally distributed. Therefore, the non-parametric Mann-Whitney U-test was used for comparison between male and female participants. Categorical variables were compared between groups using the chi-square test, or Fisher's exact test for small numbers. The level of significance was set at $\mathrm{p}<0.05$.

\section{Ethical considerations}

Ethical clearance was obtained from Institutional Review Board (IRB) from the University of Rwanda, College of Medicine and Health Sciences (CMHS) and permission was sought from the National Paralympic Committee (NPC). An information sheet was presented to the participants explaining the topic of research, the study procedures and the benefits. Participation was voluntary and consent forms were signed while parents or guardians signed for players below 18 years. The later provided also their assent to participate in the study. Participants were free to withdraw from the study at any time without explanation or any impact. The information was kept with high level of confidentiality. No names were written on the forms nor were used during data analysis and presentation.

\section{Results}

\section{Demographic characteristics}

There were 158 players that participated in the study. There were on average $9 \pm 1$ players per team. A large number of respondents were males $(n=105,66.5 \%)$ and a low number were female $(n=53,33.5 \%)$. The mean age of the players was $25 \pm 6$ years while the youngest player was 15 years old and the oldest was 47 years old. The players' mean experience was $3 \pm 3$ years, (interquartile range $=4)$. The majority of players $(n=123,78 \%)$ had $1-5$ years of experience for both males and females. MannWhitney $U$ test indicated that there was no difference in the distribution of age $(p=0.940)$, weight $(p=0.053)$ and experience $(p=0.490)$ between male and female players. The details of the demographic characteristics are shown in Table 1.

Table 1. Demographic characteristics of the participants $(n=158)$

\begin{tabular}{|c|c|c|c|c|}
\hline Variables & & Total & Male & Female \\
\hline & & $\mathbf{N}(\%)$ & $\mathrm{N}(\%)$ & $\mathbf{N}(\%)$ \\
\hline \multirow{3}{*}{ Age group (years) } & $15-25$ & 101(64) & $66(42)$ & $35(22)$ \\
\hline & $26-36$ & $49(31)$ & $35(22)$ & $14(9)$ \\
\hline & $37-47$ & $8(5)$ & $4(3)$ & $4(3)$ \\
\hline \multirow{3}{*}{ Weight (Kg) } & $40-60$ & $98(62)$ & $62(39)$ & $36(23)$ \\
\hline & $61-81$ & $58(37)$ & $43(27)$ & $15(9)$ \\
\hline & $82-102$ & $2(1)$ & 0 & $2(1)$ \\
\hline \multirow{3}{*}{ Experience (years) } & $1-5$ & $123(78)$ & $77(49)$ & $46(29)$ \\
\hline & $6-10$ & $32(20)$ & $26(16)$ & $6(4)$ \\
\hline & $11-15$ & $3(2)$ & $2(1)$ & $1(1)$ \\
\hline \multirow{2}{*}{ Marital status } & Single & $123(78)$ & $82(52)$ & $41(26)$ \\
\hline & Married & $35(22)$ & $23(15)$ & $12(8)$ \\
\hline \multirow{6}{*}{ Occupation } & Student & $63(40)$ & $41(16)$ & $22(14)$ \\
\hline & Cultivator & $27(17)$ & $17(11)$ & $10(6)$ \\
\hline & Public worker & $11(3)$ & $11(7)$ & $2(1)$ \\
\hline & Professional player & $21(13)$ & $16(10)$ & $5(3)$ \\
\hline & Business & $21(13)$ & $11(7)$ & $10(6)$ \\
\hline & Jobless & $13(8)$ & $9(6)$ & $4(3)$ \\
\hline \multirow{5}{*}{ Playing position } & Server & $39(25)$ & $24(15)$ & $15(9)$ \\
\hline & Attacker & $38(24)$ & $25(16)$ & $13(8)$ \\
\hline & Receiver & $9(6)$ & $6(4)$ & $3(2)$ \\
\hline & Defender & $59(37)$ & $39(25)$ & $20(13)$ \\
\hline & Blocker & $13(8)$ & $11(7)$ & $2(1)$ \\
\hline
\end{tabular}

Types of disability among sitting volleyball players Among 158 sitting volleyball players, 108 (68\%) had deformities while $53(32 \%)$ were amputated. The foot and ankle complex was the body part with the most prevalent deformities $(\mathrm{n}=32,20 \%)$ followed by knee joint $(n=24,15 \%)$ and the lower leg $(n=18,11 \%)$. Lower limb amputation was also observed with more below knee amputations ( $\mathrm{n}=23,14 \%$ ) followed by equal number of through knee and above knee amputation with the frequency of $11(7 \%)$ each. Eight participants represented with the upper limb deformities.

\section{Prevalence of Injuries}

Out of 158 sitting volleyball players 58 (37\%) sustained at 
least one injury. Thirty-five (22\%) players sustained one injury while $23(15 \%)$ sustained two or more injuries. Out of 105 male sitting volleyball players, 37 (35\%) sustained injuries while $21(40 \%)$ out of 53 females were injured. Forty-eight percent of injuries $(n=43)$ were mild which is consistent for both male and female. Moderate injuries constituted $25 \%$ of injuries ( $\mathrm{n}=22$ ) with proportional contribution for male and female. Recurrent injuries were also reported to represent $26 \%$ of injuries ( $n=23$ ). There was no statistical difference in training, match, severity or recurrence of injuries comparing male and female (Table 2).

Table 2. Prevalence of injuries during training and competition stratified per gender $(n=89)$

\begin{tabular}{|c|c|c|c|c|c|}
\hline Injuries & Characteristics & $\begin{array}{l}\text { Total } \\
\text { n (\%) }\end{array}$ & $\begin{array}{r}\text { Male } \\
\text { n (\%) }\end{array}$ & $\begin{array}{c}\text { Female } \\
\text { n }(\%)\end{array}$ & P-value \\
\hline Total injuries & & $89(100)$ & $60(67)$ & $29(33)$ & \\
\hline Training injuries & & $46(52)$ & $31(35)$ & $15(17)$ & 0.823 \\
\hline Match injuries & & $43(48)$ & $29(33)$ & $14(16)$ & 0.823 \\
\hline \multicolumn{6}{|c|}{ Number of Injuries per player } \\
\hline & 1 & $35(39)$ & $22(25)$ & $13(15)$ & 0.610 \\
\hline & $>1$ & $23(26)$ & $14(16)$ & $8(9)$ & 0.211 \\
\hline \multicolumn{6}{|l|}{ Severity } \\
\hline & Minimal & $20(22)$ & $16(18)$ & $4(4)$ & 0.275 \\
\hline & Mild & $43(48)$ & $29(33)$ & $14(16)$ & 0.823 \\
\hline & Moderate & $22(25)$ & $12(13)$ & $10(11)$ & 0.222 \\
\hline & Severe & $4(4)$ & $3(3)$ & $1(1)$ & 1 \\
\hline \multicolumn{6}{|l|}{ Recurrence } \\
\hline & Non-recurrent & $66(74)$ & $45(51)$ & $21(24)$ & 0.800 \\
\hline & Recurrent & $23(26)$ & $15(17)$ & $8(9)$ & 0.800 \\
\hline
\end{tabular}

\section{Severity of injuries by location and type}

The severity of injuries was classified according to the days that players were unable to participate in full training or competition. An injury that caused a time loss of 1-3 days was classified as "minimal", while injuries that resulted in an absence from training or matches for 4-7, 8-21 and more than 21 days were classified as "mild", "moderate" and "severe" injuries respectively. The days lost due to injuries were computed from the date next to the day of injury to the date of return to full participation in the game.

Upper limbs were the most affected body parts ( $n=67$, $75 \%)$ with more injuries to the fingers $(n=17)$ followed by the wrist $(n=15)$ and the hand $(n=14)$. There was no statistically significant difference in the occurrence of injuries according to the anatomical location. Strains $(n=34)$ were the most prevalent injury type followed by contusions $(n=26)$. Females sustained more dislocation injuries than males, with a statistically significant difference. All the recurrent injuries were "early recurrent" happening within two months. More than half of recurrent injuries $(52 \%)$ were moderate while mild and minimal had 22\% of injuries each. Recurrent injuries caused significantly longer median absence time than first time injuries $(\mathrm{p}=0.001)$. Table 3 shows the details of the location and type injuries according to the severity.
Table 3. Severity of injuries according to location and type $(n=89)$

\begin{tabular}{lrrrrr}
\hline & Total & \multicolumn{3}{c}{ Severity } \\
\hline Injuries & $\mathrm{n}(\%)$ & Minimal & Mild Moderate & Severe \\
Injury location & & & & & \\
Head/ face & $1(1)$ & 1 & 0 & 0 & 0 \\
Shoulder & $8(9)$ & 0 & 4 & 4 & 0 \\
Elbow & $10(11)$ & 3 & 5 & 1 & 1 \\
Wrist & $15(17)$ & 3 & 8 & 4 & 0 \\
Hand & $14(16)$ & 2 & 9 & 3 & 0 \\
Finger & $17(19)$ & 4 & 9 & 2 & 2 \\
Forearm & $3(3)$ & 1 & 1 & 1 & 0 \\
Back & $6(7)$ & 3 & 1 & 1 & 1 \\
Buttock & $6(7)$ & 1 & 3 & 2 & 0 \\
Thigh & $2(2)$ & 1 & 1 & 0 & 0 \\
Knee & $6(7)$ & 1 & 2 & 3 & 0 \\
Ankle/ foot & $1(1)$ & 0 & 0 & 1 & 0 \\
Injury type & & & & & \\
Contusion & $26(29)$ & 5 & 17 & 4 & 0 \\
Dislocation & $10(11)$ & 0 & 2 & 7 & 1 \\
Fracture & $2(2)$ & 0 & 0 & 0 & 2 \\
Laceration & $9(10)$ & 3 & 6 & 0 & 0 \\
Sprain & $34(38)$ & 9 & 15 & 10 & 0 \\
Strain & $3(3)$ & 1 & 2 & 0 & 0 \\
Other & $5(6)$ & 2 & & & \\
Recurrence & & & 1 & 1 & 1 \\
Recurrent & $23(26)$ & 5 & 5 & 12 & 1 \\
No recurrent & $66(74)$ & 15 & 38 & 10 & 3 \\
Total & 89 & 20 & 43 & 22 & 4 \\
\hline & $(100)$ & & & & \\
\hline & & & & &
\end{tabular}


Number of days lost due to injury by location and type

A total of 669 days were lost due to injuries with the worst injury causing the player to miss 54 days. Sprain constituted the highest number of injuries affecting mostly the fingers, wrists and shoulders and knees. Contusions were the second most common injury type affecting mostly hands, elbow and buttocks. The consequence of injury was also assessed by the days of play lost due to injury, and this showed that elbow injuries accounted for $23 \%$ of time lost for female players followed by the hand (14\%). For males, injuries to the fingers accounted for $29 \%$ of time loss injuries followed by the wrist (17\%) and the shoulder (14\%) (Table 4).

Table 4. Number of days lost by location and type of injuries

\begin{tabular}{|c|c|c|c|c|}
\hline \multirow{2}{*}{\multicolumn{2}{|c|}{$\begin{array}{ll}\text { Location } & \text { Type } \\
\text { Ankle/ foot } & \end{array}$}} & Frequency & Mean \pm SD & Number of days lost \\
\hline & & 1 & 8 & 8 \\
\hline & Sprain & 1 & 8 & 8 \\
\hline \multicolumn{2}{|l|}{ Back } & 6 & $9 \pm 10$ & 55 \\
\hline & Strain & 1 & 4 & 4 \\
\hline & Other & 5 & $10 \pm 11$ & 51 \\
\hline \multicolumn{2}{|l|}{ Buttock } & 6 & $7 \pm 6$ & 39 \\
\hline & Contusion & 6 & $7 \pm 6$ & 39 \\
\hline \multicolumn{2}{|l|}{ Elbow } & 10 & $9 \pm 16$ & 87 \\
\hline & Contusion & 6 & $4 \pm 3$ & 25 \\
\hline & Dislocation & 1 & 54 & 54 \\
\hline & Laceration & 2 & $2 \pm 1$ & 3 \\
\hline & Sprain & 1 & 5 & 5 \\
\hline \multicolumn{2}{|l|}{ Finger } & 17 & $9 \pm 14$ & 151 \\
\hline & Contusion & 3 & 1 & 3 \\
\hline & Dislocation & 1 & 17 & 17 \\
\hline & Fracture & 2 & $43 \pm 6$ & 86 \\
\hline & Laceration & 1 & 6 & 6 \\
\hline & Sprain & 10 & $4 \pm 3$ & 39 \\
\hline \multicolumn{2}{|l|}{ Forearm } & 3 & $10 \pm 9$ & 29 \\
\hline & Contusion & 1 & 3 & 3 \\
\hline & Dislocation & 1 & 20 & 20 \\
\hline & Sprain & 1 & 6 & 6 \\
\hline \multicolumn{2}{|l|}{ Hand } & 14 & $5 \pm 6$ & 69 \\
\hline & Contusion & 7 & $4 \pm 2$ & 25 \\
\hline & Dislocation & 2 & 18 & 36 \\
\hline & Laceration & 4 & $2 \pm 1$ & 7 \\
\hline & Strain & 1 & 1 & 1 \\
\hline \multicolumn{2}{|c|}{ Head/ face } & 1 & 7 & 7 \\
\hline & Laceration & 1 & 7 & 7 \\
\hline \multicolumn{2}{|l|}{ Knee } & 6 & $8 \pm 7$ & 50 \\
\hline & Contusion & 1 & 2 & 2 \\
\hline & Dislocation & 1 & 15 & 15 \\
\hline & Sprain & 4 & $8 \pm 7$ & 33 \\
\hline \multicolumn{2}{|l|}{ Shoulder } & 8 & $9 \pm 7$ & 69 \\
\hline & Contusion & 1 & 2 & 2 \\
\hline & Dislocation & 3 & $9 \pm 11$ & 27 \\
\hline & Sprain & 4 & $10 \pm 6$ & 40 \\
\hline \multicolumn{2}{|l|}{ Thigh } & 2 & $9 \pm 7$ & 8 \\
\hline & Laceration & 1 & 6 & 6 \\
\hline & Strain & 1 & 2 & 2 \\
\hline \multicolumn{2}{|l|}{ Wrist } & 15 & $6 \pm 7$ & 97 \\
\hline & Contusion & 1 & 1 & 1 \\
\hline & Dislocation & 1 & 18 & 18 \\
\hline & Sprain & 13 & $6 \pm 7$ & 78 \\
\hline
\end{tabular}




\section{Discussion}

\section{Characteristics of the participants}

The majority of sitting volleyball players in Rwanda are predominantly young. This is in line with the leading proportion of students. The increase in the number of youth students in the game follows the establishment of interschool paralympic competitions in Rwanda by NPC and interschool sports federation. The current study showed that most players had 1-5 years of experience; which meant that sitting volleyball is a new discipline for many players. Players in the present study are younger compared to those that participated in the study conducted by Molik and Mędasik.[11] A large number of players were male $(67 \%)$ and a low number were female $(33 \%)$. This may partly be explained by a high proportion of males with disabilities who are active compared to females in general. [12] In Rwanda, like in many other African countries, there is low participation in sports for females in general.[13] This might also be the case for females with disabilities. That is why female teams are not yet established and to facilitate inclusion the NPC decided that competing teams can use players with both gender without restriction of number. [14] This might explain the reason why no difference was observed in the demographic characteristics of the players according to gender.

The game of sitting volleyball is a sport developed for athletes mainly with lower extremity impairment for example amputations; disabling joint injuries or conditions, limb weakness. [2] This concur with the results of this study indicating that lower limb deformities and amputation were the types of disability among sitting volleyball players in Rwanda. Looking at the fact that the game is played using the upper extremities, eight players were found to have an impairment of the upper extremities. Though it could generally interfere with the performance, the players had minor deformities that could not restrict participation. The current study found that $67 \%$ of sitting volleyball players had a certain kind of deformity while $34.7 \%$ had amputation. This is in accordance with the study conducted by Molik and Mędasik[11]indicating that sitting volleyball players had predominantly amputation and lower limb paresis or poliomyelitis. However, the study conducted previously in the Hungarian sitting volleyball players indicated that the proportion of players having amputation was 39\%.[15] The difference might be found in the causes of the disability or amputation. In many African countries, the leading causes of disability are infections and communicable disease. Other causes include war, trauma, accident and congenital noninfectious diseases.[16]

\section{Injuries and severity}

The main finding of this study was that $37 \%$ of the sitting volley ball players sustained injuries while $35 \%$ of males and $40 \%$ of females incurred at least one injury. . Upper limbs represented three-quarters of injuries affecting mostly the fingers, hands and wrists. Elbow injuries accounted for $23 \%$ of time lost for female players followed by the hand $(14 \%)$. For males, injuries to the fingers accounted for $29 \%$ of time lost followed by the wrist $(17 \%)$ and the shoulder $(14 \%)$.

The results of this study indicated a higher prevalence $(37 \%)$ of sitting volleyball injuries than reported in a similar study conducted by Willick et al.[6]which found the injury prevalence of $15 \%$. These differences may be possibly explained by the fact that their sport activities are well organized and planed in terms of playing grounds, protective equipment like knee pads, ankle braces, and thigh compared to Rwandan sitting volley ball sport activities. In addition, players in paralympic games may be classified as elite and are mostly well conditioned. International players receive appropriate training that prepares the bodies to withstand the different stresses from the game.[17]However, higher percentages were found in sitting volleyball competing in major tournaments. $[10,16]$

Nearly half of injuries ( $n=43$ ) were mild which is consistent for both male and female. Moderate injuries constituted a quarter of injuries $(n=22)$ with proportional contribution for male and female. The consequence of injury was also assessed by the days of play lost due to injury, and this showed that elbow injuries accounted for $23 \%$ of time lost for female players followed by the hand $(14 \%)$. For males, injuries to the fingers accounted for $29 \%$ of time loss injuries followed by the wrist $(17 \%)$ and the shoulder $(14 \%)$. The severity of injuries in terms of days lost among male and female players was due to fracture and dislocation.

Recurrent injuries represented about a quarter of injuries and caused longer lay off time compared to first time injuries. Though it was not assessed, injuries might be poorly managed which lead to recurrence. There is therefore a need for appropriate management of injuries to reduce the rate of recurrent injuries and time lost due to injuries.

\section{Injury type and location}

There was no significant difference of the type of injuries according to gender. The present study showed that the most common type of injury was sprain that affected mostly the fingers, wrists, shoulders and knees. Similarly, the study conducted by Molik and Mędasik[11]found that the wrist and fingers were the most affected body parts $(46 \%)$ followed by the shoulder and arms (22\%). Contusions were the second most common injury type affecting mostly hands, elbow and buttocks. The authors[11] found that contusion was the most prevalent type of injury followed by abrasion, dislocation/ fracture. Contrary, sitting volleyball players competing on international level reported injuries to the back and shoulder injuries.[18] Additionally, Wieczorek et al.[7] reported that persons with dysfunction of one limb, with the other limb for play, are often exposed to the overtraining of the healthy limb. 


\section{Limitations}

Little information has been published regarding the prevalence of injury among sitting volleyball players. Detailed information regarding injuries could not be obtained because there were no health professionals in the teams. Data collection was based on self-reports that are often open to bias and misreporting. This was minimized by the weekly visits to the teams by the research personnel. As there is no guideline on data collection procedure for injuries in sports with disability, we used the consensus on injury definitions and data collection procedures used in able-bodied studies. The sample size was small which caused limitations in drawing strong conclusion.

\section{Conclusion and recommendations}

The study found a high prevalence of injuries among male and female sitting volleyball players with disability in Rwanda. The body sites prone to injuries in this study were fingers, hands and wrists. The common types of injuries were sprain followed by contusions. Nearly half of injuries were mildly and moderately severe. A quarter of injuries were recurrent and half of them were moderately severe and caused significant longer lay off time than first time injury. This study indicated that persons presenting with disability should not encounter barrier in undertaking sitting volleyball especially in a low-income country with limited resources. However, the health and integrative advantages should outweigh the risk of adverse effects associated with participation in sports such as injuries. There is therefore a need for injury prevention intervention. Further studies are also needed in this area taking into consideration individual exposure to establish risk factors for injuries.

\section{Acknowledgments}

We extend special thanks to all the sitting volleyball players that participated in this study.

\section{Conflict of interests}

There are none.

\section{Authors' contribution}

$\mathrm{AN}, \mathrm{BHN}, \mathrm{JBN}, \mathrm{AFN}$, and JS were responsible for the conceptualisation, design, data collection and analysis. $\mathrm{AN}, \mathrm{AK}$, and JBS contributed to the interpretation of the data, the writing and revisions to the manuscript. All authors approved the final version of the manuscript.

\section{Funding support \\ None}

This article is published open access under the Creative Commons Attribution-NonCommercialNoDerivatives (CC BYNC-ND4.0). People can copy and redistribute the article only for noncommercial purposes and as long as they give appropriate credit to the authors. They cannot distribute any modified material obtained by remixing, transforming or building upon this article. See https://creativecommons.org/licenses/by-nc-nd/4.0/

\section{References}

1. Vliet P Van De. Paralympic athlete's health. Br J Sports Med. 2012;46:458-9.

2. Palao J, Santos J, Urena A. Effect of team level on skill performance in volleyball. Int J Perform Anal Sport. 2012;4:50-60.

3. NPC NPC. About NPC Rwanda. 2014.

4. Barayagwiza P. Factors related to sport preferences among youth with physical disability in Rwanda. University of the Western Cape; 2011.

5. Fagher K, Lexell J. Sports-related injuries in athletes with disabilities. Scand J Med Sci Sport. 2014;24:e320-31.

6. Willick SE, Webborn N, Emery C, Blauwet CA, Pitgrosheide P, Stomphorst J, et al. The epidemiology of injuries at the London 2012 Paralympic Games. 2013;1-7.

7. Wieczorek J, Wieczorek A, Jadczak L, Sliwowski R, Pietrzak M. Physical activity and injuries and overstraining syndromes in sitting volleyball players. Stud Phys Cult Tour. 2007;14:299-305.

8. Fuller CW, Ekstrand J, Junge a, Andersen TE, Bahr $\mathrm{R}$, Dvorak J, et al. Consensus statement on injury definitions and data collection procedures in studies of football (soccer) injuries. Br J Sports Med. 2006;40:193-201.

9. Hägglund M, Waldén M, Bahr R, Ekstrand J. Methods for epidemiological study of injuries to professional football players: developing the UEFA model. Br J Sports Med. 2005;39:340-6.

10. Ardern CL, Bizzini M, Bahr R. It is time for consensus on return to play after injury : five key questions. Br J Sports Med. 2016;50:13-6.

11. Molik B, Mędasik A. Characteristic of sport injuries in team games for persons with disabilities. J Orthop Trauma Surg Relat Res. 2011;6:21-6.

12. NISR NI of S of R. the Third Integrated Household Living Conditions Survey ( Eicv3 ). 2011.

13. Brady M. Creating Safe Spaces and Building Social Assets for Young Women in the Developing World : A New Role for Sports. Women's Stud Q. 2005;33:35-49.

14. NPC NPC. Members and sports. 2010.

15. Katalin K. Injury-and sport-specific training for sportsman with disability -sitting volleyball players. PhD Thesis. Summ. Semmelweis University; 2008.

16. Cameron DL, Nixon S, Parnes P, Pidsadny M. Children with disabilities in low-income countries. Paediatr Child Health. 2005;10:269-72.

17. Zhang J, Bader D, Twycross-Lewis R, Padhiar N. A prospective cohort study of vertical pressure on hands of sitting volleyball players using tekscan pressure mat. Br J Sports Med. 2011;45:e1:3-5.

18. Mustafins P, Landor A, Vetra A, Scibrja I. Rate and type of participation limiting health disorders in sitting volleyball players (health disorders in sitting volleyball players). Pap Anthropol. 2008;17:233-47. 